\title{
Development of polyion complex vesicles (PICsomes) from block copolymers for biomedical applications
}

\begin{abstract}
Akihiro Kishimura
Polyion complex (PIC) formation is one of the most powerful techniques for obtaining molecular self-assemblies in aqueous media. The simple preparation process based on multiple electrostatic interactions is quite attractive for material syntheses, as well as biomedical applications. Therefore, it is desirable to control PIC architectures at the nanoscale in order to expand the scope of PIC materials. In this review article, recent progress on PIC vesicles (PICsomes) is summarized. PICsomes were first developed by my research group, and we recently succeeded in controlling the sizes and structural uniformity of the vesicles. Furthermore, the characteristic dynamic nature of PICs was revealed: PICs were found to exhibit reversible association/ dissociation and structural transformation. We demonstrated that crosslinking the PIC layers of PICsomes is a powerful method for tuning properties such as stability and permeability. Finally, the potential utility of PICsomes for drug delivery nanocarriers was examined, and their future biomedical application is discussed.
\end{abstract}

Polymer Journal (2013) 45, 892-897; doi:10.1038/pj.2013.33; published online 10 April 2013

Keywords: block copolymers; drug delivery system; environmentally sensitive; polyion complexes; self-assembly; vesicles

\section{INTRODUCTION}

Bottom-up nanofabrication is one of the key approaches of nanotechnology. Most bottom-up nanofabrication is achieved by self-assembly of molecules that are designed based on the principles of supramolecular chemistry. Recently, there has been an increase in the number of studies devoted to the rational design of nanostructured materials in aqueous media owing to their potential utility for biomedical applications, particularly related to optimization of the activity of materials in vivo, as well as in vitro. Several reports have pointed out the significance of detailed material properties such as sizes, shapes and morphologies, surface structures and surface chemistry, rigidity and deformability and so on. ${ }^{1-4}$

Very recently, submicron-scale materials have attracted great attention as drug nanocarriers. There are some critical requirements for such nanocarriers to overcome biological barriers, including biocompatibility, long-circulation properties and tissue permeability. ${ }^{1-7}$ Some of nano-formulations, such as Doxil, Abraxane and PEGASYS, have been approved. In particular, nano-vehicles with biocompatibility and a diameter $<200 \mathrm{~nm}$ are known to accumulate selectively in solid tumors. This phenomenon is accounted for by vascular hyperpermeability and immature lymphatic drainage, and is termed the enhanced permeability and retention effect. ${ }^{8}$ Above all, block copolymers are one of the most powerful tools for producing nanomaterials designed for biomedical applications in terms of precise control of their structure and easy improvement of their biocompatibility. ${ }^{9}, 10$ The most typical examples are amphiphilic block copolymers, which can be platforms for hydrophobic drug conjugation. Many types of formulations have been reported based on amphiphilic block copolymers, such as micelles, vesicles and emulsions. In fact, some anticancer-drug-loaded polymeric micelles are in clinical trials. However, these amphiphilic polymer selfassemblies often involve the use of organic solvents and multiple harsh steps to produce uniform nanomaterials, leading to concerns regarding damages to cargoes and toxicity in humans. Also, in the case of the delivery of water-soluble biomacromolecules, their low solubility in nonaqueous media and fragile nature limit the use of organic solvents.

To overcome these processing limitations, utilization of polyion complexes (PICs) can provide an effective solution because PIC formation can be carried out in aqueous media without any organic solvent, and the preparation process is remarkably straightforward. Typically, PICs are obtained by simply mixing oppositely charged macromolecules. There are many examples of PIC nanoparticles, such as PIC micelles and nanosized aggregates, that are designed for effective delivery of genes, small nucleic acids, proteins, charged macromolecular drugs and so on. ${ }^{11-13}$ Nevertheless, in terms of the precise control of PIC architectures on the nano-/mesoscopic scale, PIC fabrication technology is not well-developed for structures other than micelle architectures. In particular, there are far fewer examples of PIC hollow capsules in spite of their potential applications, such as protein vehicles, semipermeable capsules, environment-sensitive materials, environment-friendly materials and so on. In this review 
article, I provide an overview of research on the development of well-defined hollow PIC architectures, particularly focusing on PIC vesicles termed 'PICsomes', performed by my research group and collaborators. 'PICsomes' are the first example of polymeric vesicles spontaneously formed in aqueous media, and are characterized by their simple fabrication, semipermeability and biocompatibility. The design principles, basic properties, and potential utilities of PICsomes are briefly summarized.

\section{DESIGN OF PIC HOLLOW ARCHITECTURE: DEVELOPMENT OF PICSOMES}

There are two major approaches to building hollow PIC architecture through molecular self-assembly (Figure 1). The most popular approach is a layer-by-layer (LbL) method that was first developed by Möhwald and coworkers ${ }^{14}$ in 1998 based on homopolymer-type polyelectrolytes. In this method, $\mathrm{LbL}$ architecture is constructed on a charged surface of colloidal particles, or a template particle, through alternately immersing the template in a negatively charged polyelectrolyte solution and a positively charged solution. The biggest advantage of this method is that the membrane and surface properties are tunable. ${ }^{15}$ However, LbL hollow capsules always require template particles and multiple immersion processes, and in some cases, removal of the template is needed; these are significant concerns in terms of simple fabrication. Another approach is the direct formation of hollow architecture, or vesicle formation, based on PIC assembly. In fact, there were no reports on the spontaneous formation of PIC vesicles, or PICsomes, using block copolymers until they were developed by our group. ${ }^{16}$

Analogous to amphiphilic molecular assemblies, the molecular packing of block copolymers determines the resulting morphologies of polymer self-assemblies. This may be true for PIC systems, and thus, we tried to adjust the polymer composition to obtain PICsomes. In our studies, oppositely charged diblock copolymers consisting of a biocompatible and water-soluble poly(ethylene glycol) (PEG) segment, and charged poly(amino acid)s segment are usually used, in view of future biomedical applications, as well as their narrow

a

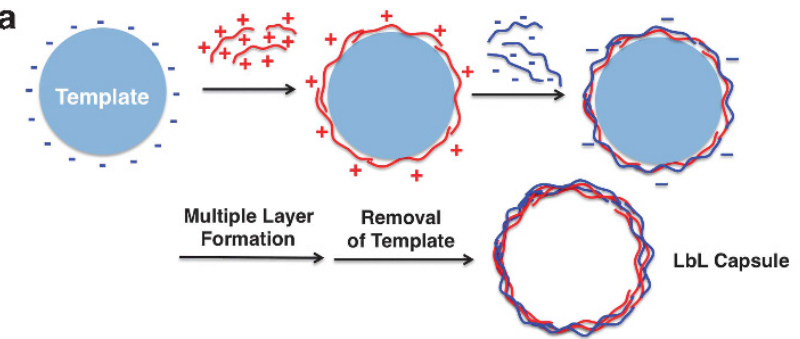

b Cationic Block Copolymers

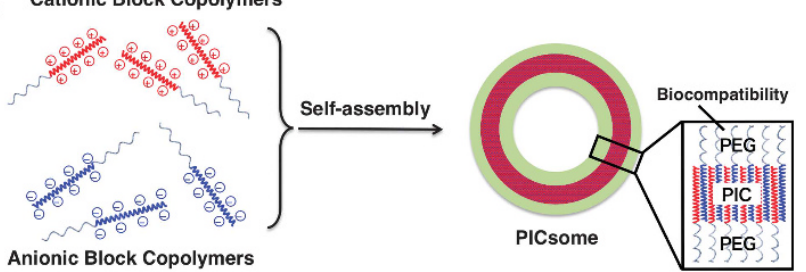

Figure 1 Schematic representations of the fabrication of polyion complex (PIC) hollow particles. (a) Layer-by-layer (LbL) capsules are obtained through alternate adsorption of oppositely charged polyelectrolytes. (b) Typically, PICsomes are prepared from oppositely charged block copolymers. molecular weight distribution (Figure 2). Typically, the diblock copolymers are mixed to neutralize their charges: that is, the primary amino groups on the polycations and the carboxylic acid moieties on the polyanions form ion pairs to give PICs. As shown in Figure 3, a large volume of PEGs, or higher PEG weight fraction $\left(f_{\mathrm{PEG}}\right)$, prevents the growth of PIC and drives micelle formation due to the immiscibility of a PEG part and PIC part, as well as large steric hindrance of PEG strands. Therefore, a small volume of PEGs, or lower $f_{\mathrm{PEG}}$, is considered to be favorable for vesicle formation, which requires expansion of PIC architecture in the two dimensional lamellar structure. ${ }^{17-19}$ To reduce $f_{\text {PEG }}$, relatively short PEG chains $(M \mathrm{w}=2000)$ were chosen for PIC formation. First, we found successful formation of PICsomes with sizes of several micrometers under the physiological salt concentration $(150 \mathrm{~mm} \mathrm{NaCl}) .{ }^{9}$ As we expected, PICsomes can be prepared in a simple manner by mixing a pair of oppositely charged polymers for a couple of minutes to neutralize their charges. Thus obtained micron-sized particles, which are called 'the first generation PICsomes' (1st-G PICsomes) in this article, are not realistic for systemic delivery of therapeutic

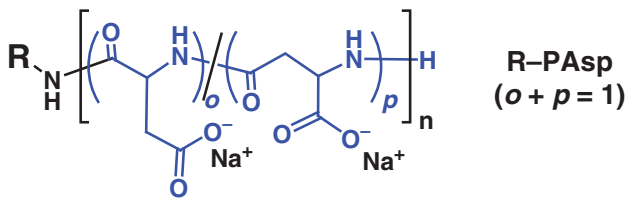

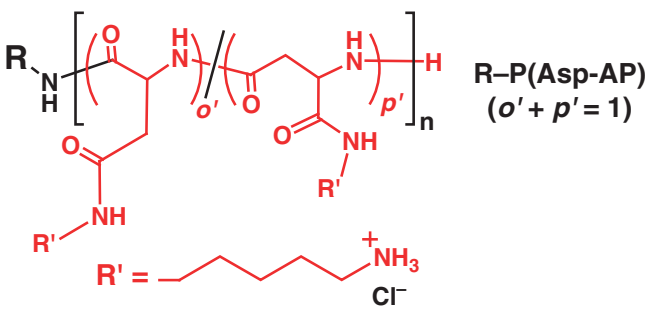<smiles>[R]C(=C)CCCCC(C)COC</smiles>

Figure 2 Chemical structures of charged polymers for polyion complex vesicles. Charged polymers are synthesized as a block copolymer bearing a single poly(ethylene glycol) (PEG) chain (Mn 2000) or a homopolymer.

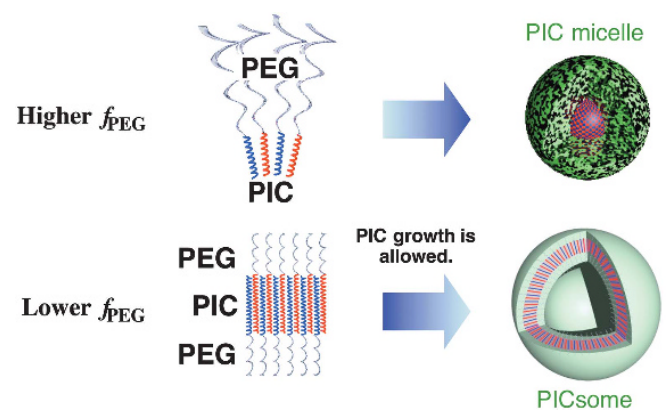

Figure 3 Schematic representations of the structural control of polyion complex (PIC) assemblies. Larger poly(ethylene glycol) (PEG) volume inhibits the increase of the association number of polymers mainly because of steric hindrance of PEG chains. 
compounds because of their large size. Nonetheless, from a fundamental viewpoint, some significant information was obtained through direct observation by using confocal laser scanning microscopy. As a result, we confirmed direct loading of watersoluble macromolecules such as dextrans, proteins and so on, during the PIC formation process. Notably, this gentle process enables encapsulation of proteins into PICsomes, preserving the proteins' function (Figure 4). ${ }^{20}$ confocal laser scanning microscopy observation also clarified the semipermeability of PICsomes, which is a characteristic property that is not observed for conventional vesicles consisting of amphiphilic molecules. In fact, large molecules were negligibly released from inside of the PICsomes and they could not penetrate the PICsomes from the exterior, while small compounds and ions easily passed through the PIC membranes. Moreover, encapsulated proteins were protected from attack by proteinases within the PICsomes. ${ }^{20}$ These results indicate that PICsomes are much more suitable for protein vehicles and building nanoreactors on the basis of selective permeability to small compounds than are conventional hollow architectures consisting of amphiphilic molecules or requiring templates for fabrication.

Making the structures of the PICsomes uniform and controlling their sizes are challenging issues as a matter of engineering, and are essential for future applications to nanomedicine. Unfortunately, the size is not controllable and the structure is not simple in the case of the 1st-G PICsomes. In the meantime, we found that $f_{\mathrm{PEG}}<10 \%$ is favorable for vesicle formation. ${ }^{17,18}$ It is noteworthy that detachment of PEG chains from the PIC micelles induced structural transformation to submicron-sized PIC vesicles when $f_{\mathrm{PEG}}$ became lower than $\sim 10 \%{ }^{17}$ This result also indicates that use of homopolymers is possibly effective for vesicle formation. Thus, we attempted PIC formation using homopolymers at $f_{\mathrm{PEG}}$ values $\leqslant 10 \%$, that is, by reducing $f_{\mathrm{PEG}}$. For this purpose, homo-P(Asp-AP) was synthesized and exploited for PIC formation (Figure 2). ${ }^{18}$ Remarkably, unilamellar vesicles with the size of $\sim 100 \mathrm{~nm}$ and narrow size distribution were directly obtained after mixing solutions of homo-P(Asp-AP) and PEG- $b$-PAsp $\left(f_{\mathrm{PEG}} \sim 8 \%\right)$ in the absence of $\mathrm{NaCl}$ when the total polymer concentration was $1 \mathrm{mg} \mathrm{ml}^{-1}$ (Figure 5). Cryo-transmission electron microscopy observation clearly illustrates hollow architecture of resulted vesicles. The thickness of the vesicle wall is $10-15 \mathrm{~nm}$, which is less than the fully extended chain length of the constituent polyelectrolyte strands $(\sim 18 \mathrm{~nm})$, roughly

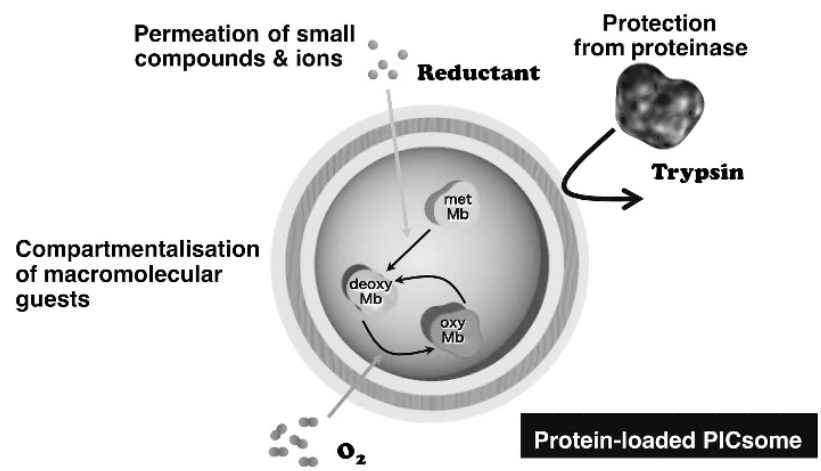

Figure 4 Schematic representation of the characteristic properties of PICsomes mainly focusing on a protein-loaded product. PICsomes encapsulate myoglobin preserving its activity and protecting the protein from enzyme attack. Partially reproduced from Kishimura et al. ${ }^{20}$ with permission. Copyright (2007) Wiley-VCH Verlag GmbH \& Co. KGaA, Weinheim. A full color version of this figure is available at Polymer Journal online. estimated by summation of the average backbone length unit (C-C-N, $0.24 \mathrm{~nm}) .{ }^{18}$ This result suggests that the vesicle wall is composed of a monolayer of PIC sandwiched by PEG palisades. Such structural regularity, or PEG-PIC-PEG three-layered structure, might be ascribed to alignment of molecular junction of block copolymers on the basis of phase separation of a PEG part and PIC part. ${ }^{21}$ More importantly, size was tunable over the range of $100-400 \mathrm{~nm}$ by changing the total polymer concentration. These submicron-sized PICsomes, that is, the second generation PICsomes, were termed 'Nano-PICsomes'. It is noteworthy that there is a linear relationship between the surface area of the Nano-PICsomes and the total polymer concentration (Figure 5). This fact indicates that the polymer concentration definitely determines the number of molecules contained in a single PICsome, which is consistent with the unilamellar structure of PICsomes. Very recently, we found that unit PIC (uPIC), which consists of a single pair of a homo-P(Asp-AP) molecule and PEG- $b$-PAsp molecule, is an elemental component of our PIC system. ${ }^{19}$ In fact, PICsomes underwent fragmentation into uPICs in response to mechanical stress generated by vortex mixing, and formed again after removal of the stress. Thus, the total polymer concentration dependency of PICsome sizes can be interpreted as uPIC-concentration dependency; in other words, the size of PICsomes can be described as a function of uPIC-concentration. Moreover, we can increase the size of PICsomes by addition of the specific amount of uPICs to pre-formed PICsomes. ${ }^{19}$ This result also support the idea that a uPIC has a molecularly frustrated state caused by immiscible nature of PEG chains with PIC domains, and a energetically unfavorable state due to relatively hydrophobic nature of PIC domains. Therefore, uPIC is active for insertion into PICsomes. This dynamic behavior might be unique for our PIC system unlike molecular assemblies composed of usual amphiphilic molecules.
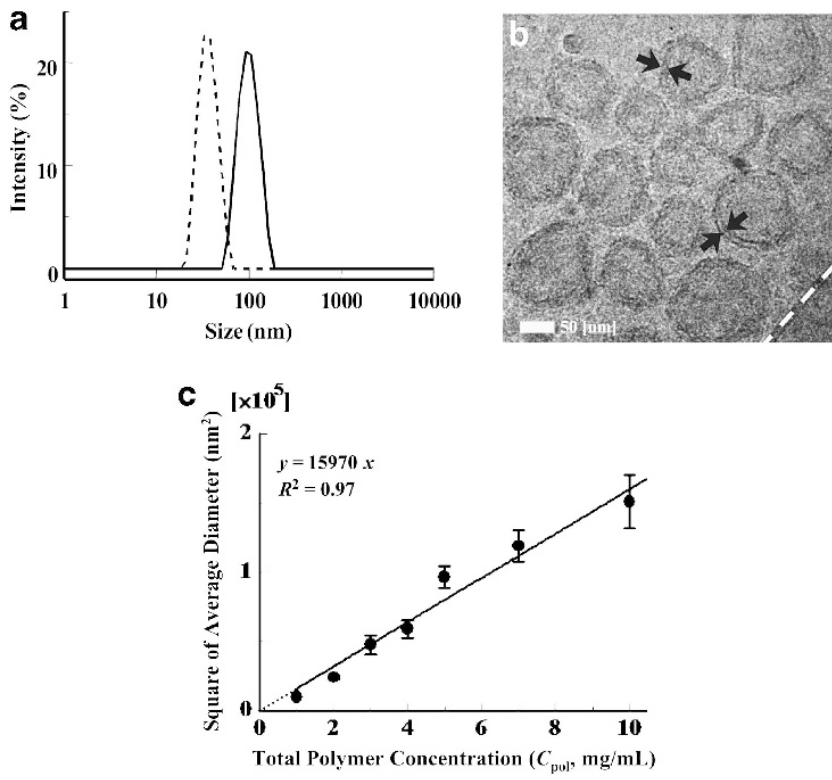

Figure 5 Formation of nanosized PICsomes (Nano-PICsomes). (a) Size distribution of PIC particles prepared at $f_{\mathrm{PEG}} \sim 15 \%$ (dashed line) and $f_{\mathrm{PEG}} \sim 8 \%$ (solid line) determined by dynamic light scattering. (b) Cryogenic phase-contrast transmission electron microscopic image of Nano-PICsomes obtained at a total polymer concentration of $1 \mathrm{mg} \mathrm{ml}^{-1}$. (c) Relationship between total polymer concentration and the square of the average diameters of the Nano-PICsomes. Partially reproduced from Anraku et al. ${ }^{18}$ with permission. Copyright (2010) American Chemical Society. 


\section{DYNAMIC RESPONSES OF PICSOMES UTILIZING THE ENVIRONMENTAL SENSITIVITY OF THE PIC SYSTEM}

Another important aspect of PIC systems is their potential sensitivity to many physical factors that determine solution properties such as $\mathrm{pH}$, ionic strength, temperature and so on. Smart or intelligent properties can be built into the PIC systems by full utilization of environmental sensitivity. Some impressive results have been reported regarding both LbL capsules and PICsomes. For example, the $\mathrm{pH}$ responsive behaviors of LbL capsules were discussed for PIC systems consisting of weak bases or acids, and unique properties such as reversible swelling/deswelling, controllable permeability and so on, were reported..$^{22,23}$ Similar $\mathrm{pH}$-sensitive properties were found in the 1st-G PICsomes based on the pH-sensitivity of weak acids, or poly(aspartic acid)s. In fact, the 1st-G PICsomes fragmented into smaller particles under conditions of low $\mathrm{pH}(\mathrm{pH} \sim 3.8)$ and reformation took place after subsequent neutralisation (Figure 6). ${ }^{24}$ Thus, 1st-G PICsomes show reversible morphological change in this $\mathrm{pH}$ range. Furthermore, 1st-G PICsomes can encapsulate macromolecules during the regeneration process. Also, changes in the permeability of PICsomes were observed in response to decreased $\mathrm{pH}$. At $\mathrm{pH} \sim 5.8$, fluorescent-labelled dextran $(\mathrm{Mn}=70000)$ can permeate PICsomes, but it does not permeate in the $\mathrm{pH}$ range between 6.2 and 7.4. These $\mathrm{pH}$-sensitive properties are promising for future application of PICsomes in the field of nanomedicine.

From the viewpoint of structural transition, we found very unique behavior of our PIC system. PICs prepared from ternary constituents, or PEG- $b$-PAsp, PEG- $b$-P(Asp-AP), and homo-P(Asp-AP), form coacervate-like dense particles in the presence of 75 and $150 \mathrm{~mm}$ $\mathrm{NaCl}$. The coacervate-like PICs obtained at $75 \mathrm{~mm} \mathrm{NaCl}$ show morphological transformation into micrometre-sized unilamellar PICsomes triggered by the local heating of individual PICs using a focused infrared laser beam (optical tweezers) ${ }^{25}$ During irradiation with the infrared beam, a PIC particle gradually transformed into a vesicular structure, but the resulting vesicle returned to the initial coacervate-like state after laser irradiation was stopped (Figure 7). In contrast, a similar transformation was not observed using PICs obtained at $150 \mathrm{~mm} \mathrm{NaCl}$, indicating that an excessive amount of $\mathrm{NaCl}$ is not favorable for vesicle formation. It should be noted that
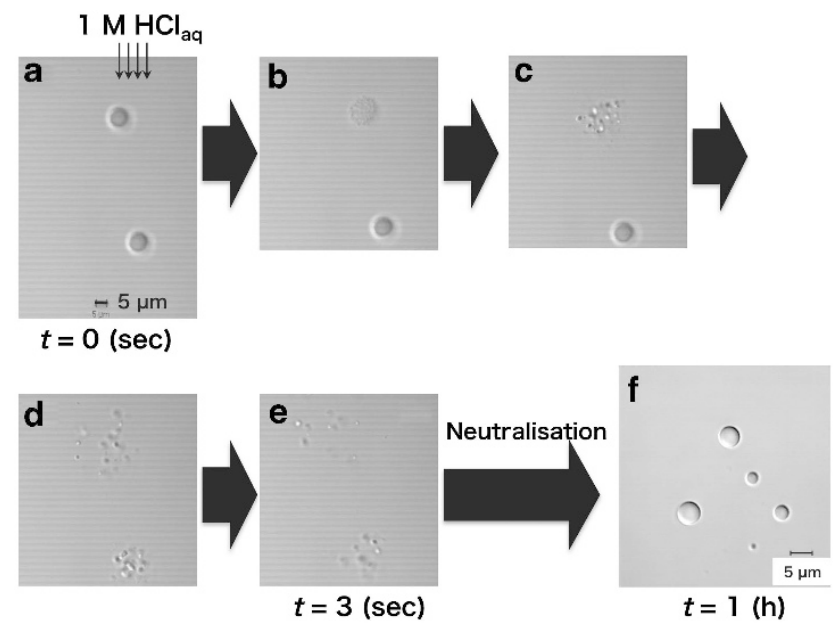

Figure 6 Reversible structural transition of PICsomes in response to $\mathrm{pH}$. (a-e) Real-time observation of PICsomes when $\mathrm{pH}$ decreased from 7.4-3.8. The arrows on the first picture show the diffusion direction of the added $\mathrm{HCl}$. (f) An optical micrograph of regenerated PICsomes $1 \mathrm{~h}$ after neutralisation. Partially reproduced from Kishimura et al. ${ }^{24}$ Copyright (2009) The Royal Society of Chemistry. the size of the formed unilamellar vesicles is evidently determined by the initial size of the coacervate-like PICs, supporting the observation that the obtained PIC membranes of the giant PICsomes have uniform structures. This nontrivial vesicle production system will lead to the development of a novel microcapsule production method performed under conditions that are gentler and much closer to physiological conditions.

\section{TUNING PROPERTIES OF PICSOMES BY CROSSLINKING}

The dynamic nature of PICsomes is not always beneficial for practical use. Actually, in the presence of $\mathrm{NaCl}$, the Nano-PICsomes lost wellcontrolled size distribution and were transformed into micron-sized particles similar to the 1 st-G PICsomes or coacervate-like products. To maintain their original structure under physiological conditions, crosslinking of PICs is quite promising. In the case of the abovementioned PICsomes, the constituent polycations have primary amino groups and the polyanions have carboxylic acids as pendant groups, and they form ion pairs. Therefore, the crosslinking reaction using 1-ethyl-3-(3-dimethylaminopropyl)carbodiimide hydrochloride effectively proceeded. After treatment with an excess amount of ethyl3-(3-dimethylaminopropyl)carbodiimide hydrochloride, the resulting products showed sufficient stability, that is, no size distribution change, even under physiological conditions. ${ }^{18}$ Also, cryogenic transmission electron microscope observations revealed that the unilamellar structure was still preserved after crosslinking. In addition, the permeability of the PIC membrane can be controlled by crosslinking. Actually, FITC-dextran $(M n=10000)$ was slowly released from inside of the Nano-PICsomes before crosslinking. In contrast, excessively crosslinked products showed significantly suppressed release of dextran. Considering the abovementioned smart response, two potentially effective approaches for advanced controlled-release systems are a moderate degree of crosslinking and the development of stimuli-sensitive de-crosslinking.

\section{IN VIVO APPLICATION OF CROSSLINKED NANO-PICSOMES}

It is essential for nanoparticles to be equipped with multiple tunable properties in order to build versatile platforms for nanomedicine. In that sense, PICsomes appear to be useful owing to their potentially tunable properties such as size, permeability, dynamic behavior, deformability and so on.

For full utilization of the size tunability of PICsomes, we carefully examined the biodistribution of crosslinked Nano-PICsomes with different sizes in C26 tumor-bearing mice. ${ }^{26}$ Plasma clearance results indicate that all of the Nano-PICsomes show good longevity in the blood stream regardless of their sizes (mean residence time $>40 \mathrm{~h}$ ). Specifically, PICsomes with diameters in the range of $150-200 \mathrm{~nm}$ showed prolonged blood circulation (mean residence time $\sim 70 \mathrm{~h}$ ). It is noteworthy that PICsomes larger than $200 \mathrm{~nm}$ in diameter still showed excellent blood circulation, while particulate formulations with sizes $>200 \mathrm{~nm}$ typically do not pass through fenestrations in the spleen and result in shorter circulation. ${ }^{1,27}$

The critical size of nanocarriers $(\sim 150 \mathrm{~nm})$ discriminating the permeability into normal and tumor tissues was determined using long-circulating Nano-PICsomes (Figure 8). In the C26 tumor tissue, PICsomes $\geqslant 150 \mathrm{~nm}$ show trivial accumulation after $240 \mathrm{~h}$. Also, faster accumulation took place as the size of the particles decreased. These results are clearly consistent with the mechanism known as the enhanced permeability and retention effect, ${ }^{8}$ which has often been found for macromolecular and particulate nanocarrier systems. Our results clearly confirm the critical cutoff size of particles exhibiting the enhanced permeability and retention effect for the 

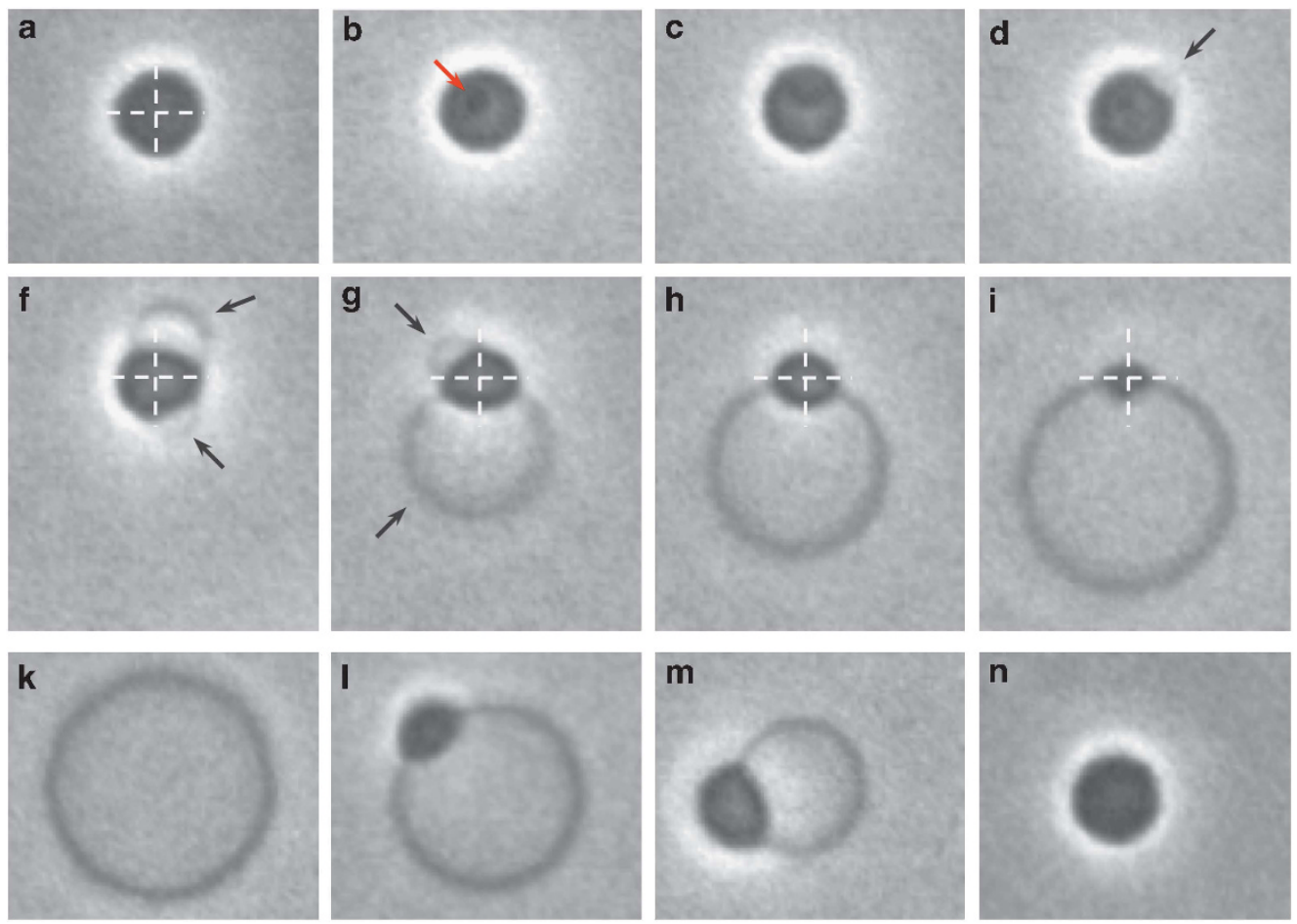

$80 \mathrm{~s}$

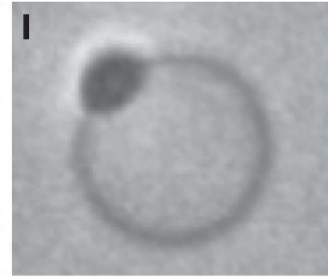

$4 \mathrm{~min}$.

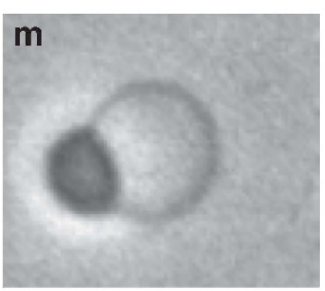

$7 \mathrm{~min}$.

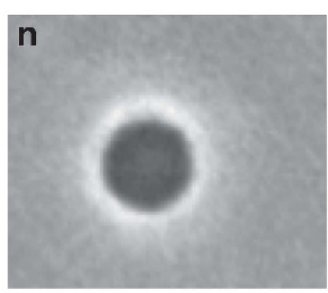

$8.5 \mathrm{~min}$.
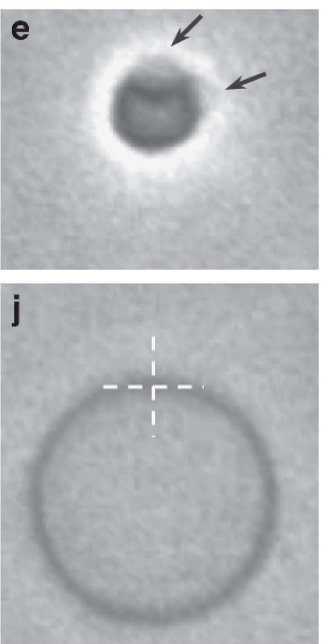

$5 \mu \mathrm{m}$

Figure 7 Time development of the morphological change of a polyion complex (PIC) droplet. (a-j) During laser irradiation; (k-n), after irradiation. Images were obtained at the $\mathrm{NaCl}$ concentration of $75 \mathrm{~mm}$ and radiated laser power of $0.25 \mathrm{~W}$. The time of image (a) is set as $0 \mathrm{~s}$, and the elapsed times are 2.5 (b), 3.5 (c), 9.0 (d), 12 (e), 26 (f), 65 (g), 96 (h), 140 (i) and $165 \mathrm{~s}$ (j). The bottom images (k-n) show the time course of the morphological change after laser irradiation was stopped. The intersections of the two white dashed lines indicate the position of the laser spot (a, $\mathbf{f}-\mathbf{j})$. The red arrow indicates a dark spot (b) and the black arrows indicate individual vesicular compartments (d-g). Partially reproduced from Oana et al. ${ }^{25}$ with permission. Copyright (2009) Wiley-VCH Verlag GmbH \& Co. KGaA, Weinheim.
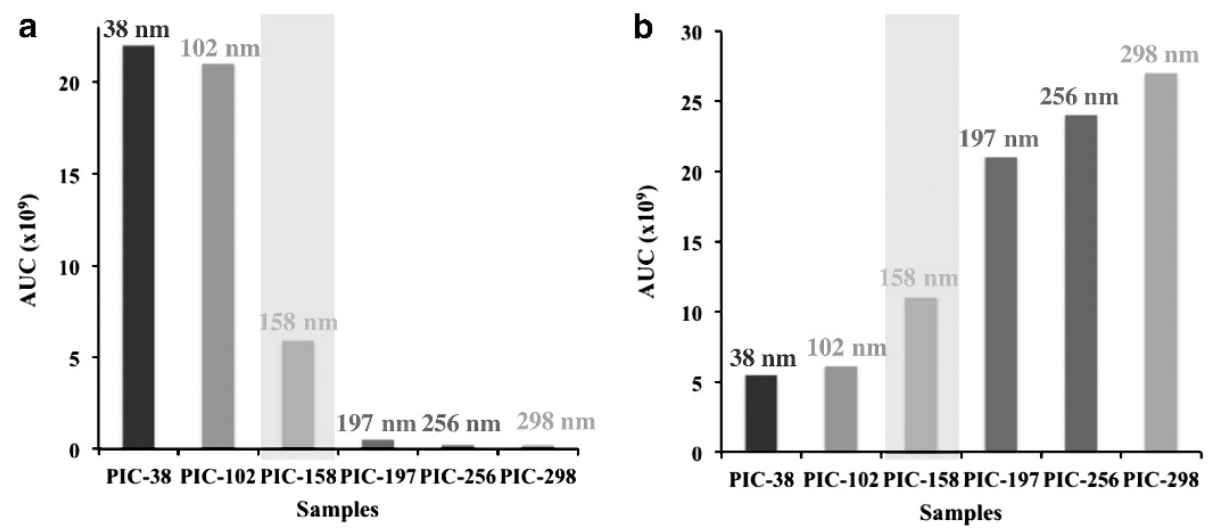

Figure 8 Accumulation of polyion complex (PIC) nanoparticles in tumors and the spleen. The area under the curves of crosslinked Nano-PICsomes (red: PIC-102, orange: PIC-158, green: PIC-197, blue: PIC-256, pink: PIC-298) and PIC micelles (black: PIC-38) in a C26 tumor (a) and the spleen (b). PIC-X stands for PIC particles with a diameter of X nm. Partially reproduced from Anraku et al. ${ }^{26}$ Copyright (2011) The Royal Society of Chemistry. A full color version of this figure is available at Polymer Journal online.

tumor model used for this experiment. The opposite trend is observed in spleen accumulation. PICsomes $>150 \mathrm{~nm}$ underwent significant spleen uptake over $240 \mathrm{~h}$. These findings related to the sizes of the particles obviously show that tuning the size of the particles is crucial to the design of nanocarriers, even when particles have biocompatibility. In addition, from some preliminary results, we found that the degree of crosslinking influences PICsome biodistribution in mice. Further investigation and discussion of this point is required for biomedical applications, and detailed results will be reported in the near future.
Materials with many tunable properties, such as PICsomes, enable us to clarify the physical and chemical properties of diseased parts of the body at the nanoscopic level, which can be defined as 'nanophysiology'. Nanophysiology can provide much insight into unresolved issues found in intractable diseases and aid in the development of novel therapeutic methods based on nanotechnology. Very recently, we confirmed encapsulation of some inorganic nanoparticles for bioimaging and successfully achieved real-time imaging using living animals. ${ }^{28}$ Thus, these PICsomes are considered to be useful tools for nanophysiology research. 


\section{SUMMARY AND OUTLOOK}

As described above, we successfully prepared the first examples of PICsomes and clarified their characteristic properties from both fundamental and practical viewpoints. Size-tunable unilamellar vesicles (Nano-PICsomes) were developed and have been exploited for use as therapeutic and diagnostic nanocarriers. Direct preparation of hollow capsules in aqueous media may widen the scope of the nano-/microencapsulation of bioactive materials, and further investigation of full utilization of tunable physical and chemical properties of PICsomes for biomedical applications is ongoing. For example, enzyme-loaded PICsomes are effective for building nano-bioreactors, based on their semipermeability and the ease of protein encapsulation, as well as their long half-life in the bloodstream.

In addition, we found that the PIC system exhibits a dynamic response to some external stimuli, indicating that the PIC system is quite useful for the design of 'smart' materials or sensing materials. However, there are many unclear aspects of the mechanism of the dynamic nature of PICs. In particular, coacervate-like dense particles are an attractive research target for cytomimetic chemistry and soft matter, but the field is still undeveloped. Our preliminary findings show that coacervate-like microparticles sometimes show a discrete nanoscopic structure within the particles, indicating that the PIC technique is powerful and versatile for constructing nanoarchitectures via simple methods. Simple preparation of the precisely controlled PIC nanoarchitectures will broaden the future utility of the PIC technique in many industrial fields owing to its low-cost, low-toxic and environmentally friendly fabrication process.

\section{ACKNOWLEDGEMENTS}

This research was supported in part by a Grant-in-Aid for Scientific Research (No. 23106705, 23685037) from the Ministry of Education, Culture, Sports, Science and Technology (MEXT) of Japan. I am deeply indebted to Professor Kazunori Kataoka (The University of Tokyo) for his continuous encouragement and discussion throughout this work. Most of this study was conducted by our students and coworkers. I am grateful to many of my coworkers, particularly Drs Hidehiro Oana and Yasutaka Anraku (The University of Tokyo), and my students.

1 Petros, R. B. \& DeSimone, J. M. Strategies in the design of nanoparticles for therapeutic applications. Nat. Rev. Drug Discovery 9, 615-627 (2010).

2 Hubbell, J. A., Thomas, S. N. \& Swartz, M. A. Materials engineering for immunomodulation. Nature 462, 449-460 (2009).

3 Alexis, F., Pridgen, E., Molnar, L. K. \& Farokhzad, O. C. Factors affecting the clearance and biodistribution of polymeric nanoparticles. Mol. Pharmaceutics 5, 505515 (2008)

4 Cabral, H., Nishiyama, N. \& Kataoka, K. Supramolecular nanodevices: from design validation to theranostic nanomedicine. Acc. Chem. Res. 44, 999-1008 (2011).

5 Peer, D., Karp, J. M., Hong, S., Farokhzad, O. C., Margalit, R. \& Langer, R. Nanocarriers as an emerging platform for cancer therapy. Nat. Nanotechnol. 2, 751-760 (2007).

6 Ferrari, M. Cancer nanotechnology: opportunities and challenges. Nat. Rev. Cancer 5 , 161-171 (2005).
7 Davis, M. E., Chen, Z. \& Shin, D. Nanoparticle therapeutics: an emerging treatment modality for cancer. Nat. Rev. Drug Discovery 7, 771-782 (2008).

8 Matsumura, Y. \& Maeda, H. A new concept for macromolecular therapeutics in cancer chemotherapy: mechanism of tumouritropic accumulation of proteins and the antitumour agent SMANCS. Cancer Res. 46, 6387-6392 (1986).

9 Kataoka, K., Harada, A. \& Nagasaki, Y. Block copolymer micelles for drug delivery: design characterization and biological significance. Adv. Drug Delivery Rev. 47, 113-131 (2001).

10 Nishiyama, N. \& Kataoka, K. Nanostructured devices based on block copolymer assemblies for drug delivery: designing structures for enhanced drug function. Adv. Polym. Sci. 193, 67-101 (2006).

11 Hartig, S. M., Greene, R. R., Dikov, M. M., Prokop, A. \& Davidson, J. M. Multifunctional nanoparticulate polyelectrolyte complexes. Pharm. Res. 24, 2353-2369 (2007).

12 Miyata, K., Nishiyama, N. \& Kataoka, K. Rational design of smart supramolecular assemblies for gene delivery: chemical challenges in the creation of artificial viruses. Chem. Soc. Rev. 41, 2562-2574 (2012).

13 Lee, Y. \& Kataoka, K. Biosignal-sensitive polyion complex micelles for the delivery of biopharmaceuticals. Soft Matter 5, 3810-3817 (2009).

14 Donath, E., Sukhorukov, G. S. M., Caruso, F., Davis, S. A. \& Möhwald, H. Novel hollow polymer shells by colloid-templated assembly of polyelectrolytes. Angew. Chem. Int. Ed. 37, 2201-2205 (1998).

15 Becker, A. L., Johnston, A. P. R. \& Caruso, F. Layer-by-layer-assembled capsules and films for therapeutic delivery. Small 6, 1836-1852 (2010).

16 Koide, Y., Kishimura, A., Osada, K., Jang, W. -D., Yamasaki, Y. \& Kataoka, K. Semipermeable Polymer vesicle (picsome) self-assembled in aqueous medium from a pair of oppositely charged block copolymers: physiologically stable micro-/nanocontainers of water-soluble macromolecules. J. Am. Chem. Soc. 128, 5988-5989 (2006).

17 Dong, W. -F., Kishimura, A., Anraku, Y., Chuanoi, S., Yamasaki, Y. \& Kataoka, K. Monodispersed Polymeric Nanocapsules: spontaneous evolution and morphology transition from reducible hetero-PEG picmicelles by controlled degradation. J. Am. Chem. Soc. 131, 3804-3805 (2009).

18 Anraku, Y., Kishimura, A., Oba, M., Yamasaki, Y. \& Kataoka, K. spontaneous formation of nanosized unilamellar polyion complex vesicles with tunable size and properties. J. Am. Chem. Soc. 132, 1631-1636 (2010).

19 Anraku, Y., Kishimura, A., Yamasaki, Y. \& Kataoka, K. Living unimodal growth of polyion complex vesicles via two-dimensional supramolecular polymerization. J. Am. Chem. Soc. 135, 1423-1429 (2013).

20 Kishimura, A., Koide, A., Osada, K., Yamasaki, Y. \& Kataoka, K. Self-assembled encapsulation of myoglobin into pegylated polyion complex vesicles (picsomes) made from a pair of oppositely charged block ionomers: a physiologically available oxygen carrier. Angew. Chem. Int. Ed 46, 6085-6088 (2007).

21 Harada, A. \& Kataoka, K. Chain length recognition: core-shell supramolecular assembly from oppositely charged block copolymers. Science 283, 65-67 (1999).

22 Mauser, T., Déjugnat, C. \& Sukhorukov, G. B. Reversible pH-dependent properties of multilayer microcapusles made of weak polyelectrolytes. Macromol. Rapid Commun. 25, 1781-1785 (2004).

23 Antipov, A. A. \& Sukhorukov, G. B. Polyelectrolyte multilayer capsules as vehicles with tunable permeability. Adv. Colloid Interface Sci. 111, 49-61 (2004).

24 Kishimura, A., Liamsuwan, S., Matsuda, H., Dong, W.-F., Osada, K., Yamasaki, Y. \& Kataoka, K. pH-dependent permeability change and reversible structural transition of PEGylated polyion complex vesicles (PICsomes) in aqueous media. Soft Matter 5, 529-532 (2009).

25 Oana, H., Kishimura, A., Yonehara, K., Yamasaki, Y., Washizu, M. \& Kataoka, K. Spontaneous formation of giant unilamellar vesicles from microdroplets of polyion complex via thermally induced phase separation. Angew. Chem. Int. Ed. 48, 4613-4616 (2009).

26 Anraku, Y., Kishimura, A., Kobayashi, A., Oba, M. \& Kataoka, K. Size-controlled longcirculating PICsome as ruler to measure critical cut-off disposition size into normal and tumor tissues. Chem. Commun. 47, 6054-6056 (2011).

27 Bertrand, N. \& Leroux, J. -C. The journey of a drug-carrier in the body: an anatomophysiological perspective. J. Control. Release 161, 152-163 (2012).

28 Kokuryo, D., Anraku, Y., Kishimura, A., Tanaka, S., Kano, M. R., Kershaw, J., Nishiyama, N., Saga, T., Aoki, I. \& Kataoka, K. SPIO-PICsomes: development of a highly sensitive and stealth-capable MRI nano-agent for tumor detection using SPIOloaded unilamellar polyion complex vesicles (PICsomes). J. Controlled Release. doi:10.1016/j.jconrel.2013.03.016 (in the press).

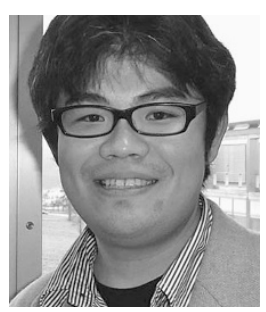

Dr Akihiro Kishimura was born in Kanagawa Prefecture, Japan in 1977. He received his B.S. degree under the supervision of Professor Masanobu Hidai from the University of Tokyo in 2000, and his PhD degree of engineering under the supervision of Professor Takuzo Aida from the University of Tokyo in 2005. He joined the research group of Professor Kazunori Kataoka in 2005, and has been an assistant professor in Department of Materials Engineering, Graduate School of Engineering, The University of Tokyo since 2006. He was a recipient of the Award for Encouragement of Research in Polymer Science from The Society of Polymer Science, Japan (2010), the Takagi award at the 20th Inteligent Materials/Systems Symposium, the best presentation award at the 18th Polymer Material Forum (2009) and several other awards. His main research interest relates to synthesis and analysis of polymeric nano-/micro-structures based on supramolecular approaches particularly in aqueous media, and application of polymeric nano-materials to the biomedical uses. 